\title{
Cameroonian Preadolescents' Perspectives of an HIV Prevention Intervention
}

\author{
Comfort Enah, Linda Moneyham, Gwendolyn Childs, Carrie Ann Gakumo
}

School of Nursing, University of Alabama, Birmingham, USA.

Email: enahcc@uab.edu

Received April 30th, 2011; Revised May 20" ${ }^{\text {th }}$ 2011; Accepted May 27 2011.

\begin{abstract}
Purpose: Determining and incorporating the perspective of the target population is important to the developments of effective and sustainable HIV prevention efforts. Cameroonian preadolescent females remain at high risk for contracting HIV and have not benefitted from targeted HIV prevention efforts. In this report, findings are presented from data collected from 60 Cameroonian preadolescent females; ages 10 - 12 years; following their completion of an HIV prevention intervention. Methods: The theory-based intervention utilized the World Health Organization's Responsible Behavior: Delaying Sex curriculum which had been tailored for the target population with the assistance of Cameroonian experts. The data reported here were collected as part of a questionnaire participants completed post-intervention. Results: With only a few exceptions, participants perceived the intervention to be appropriate and relevant. No significant differences were found in participants' assessment of the intervention across different ages and ethnic groups. Discussion: Evaluating the appropriateness of interventions to the developmental level, gender, and culture of participants is an essential step in developing effective and sustainable interventions. Findings in this study are encouraging and indicate that interventions adapted with the assistance of local experts can be perceived relevant and appropriate by participants.
\end{abstract}

Keywords: HIV Prevention, Adolescent Health, Intervention Research, Participants' Perspectives, Cameroon, Africa

\section{Introduction}

According to the 2009 AIDS epidemic update by the Joint programs on AIDS [1], sub-Saharan Africa remains the most heavily affected region in the world, accounting for over $68 \%$ of new infection in recent years. In addition, women and girls in sub-Saharan Africa continue to be disproportionately affected by HIV/AIDS [1]. While tremendous strides have been made in improving access to HIV treatment, the epidemic continues to outpace the response. Although there are numerous evidence-based prevention interventions with demonstrated effectiveness, most have been developed in western countries with cultures and other characteristics distinctly different from sub-Saharan Africa. In addition, few HIV prevention interventions have been designed specifically for young adolescent females.

In recent years, considerable progress has been made in identifying factors that contribute to the transmission of the disease in countries that are disproportionately affected by HIV/AIDS such as Cameroon, Africa. Sev- eral approaches have been used in Sub-Saharan Africa in an attempt to decrease HIV infection rates in adolescents. Included in these strategies are peer education, social marketing, mass media awareness campaigns, voluntary testing and counseling, and school-based programs [2]. School-based approaches are often advocated because of the ability to reach large numbers of adolescents in a short period of time in an environment suitable for learning and evaluation. It is generally recommended that school-based primary disease prevention interventions should begin at the primary school level because school attendance drops dramatically in most countries in this region as students transition from primary, secondary, high school and college levels [3]. The focus of HIV prevention efforts on primary school students is limited, however, by the "one size fits all" approach to interventions targeting adolescents. Little consideration has been given to the developmental differences of younger and older adolescents, as well as their level of engagement in sexual activity. Key to intervention effectiveness is the match between the intervention and the target population. 
As part of a larger study to assess the potential efficacy of an HIV prevention intervention tailored for use with Cameroonian preadolescents [4,5] we explored the acceptability and relevance of the intervention to primary school age Cameroonian girls.

The magnitude of the impact of AIDS in Sub-Saharan Africa is unparalleled in human history. In this region of the world, the disease will cripple the economies of many countries if urgent preventative interventions are not implemented $[1,6]$. The disease is a threat to people in the most productive phases of their lives, with devastating effects on families and entire cultures and populations [6]. In Cameroon, a third of those who are infected are aged 14 - 19 [7]. Among young people, the full impact of AIDS is hidden by the lengthy incubation period of the disease. Those diagnosed in young adulthood were likely infected during their adolescent and preadolescent years [8]. There is growing recognition of the need to target young adolescents and preadolescents for HIV prevention efforts prior to their engagement in sexual activity $[9,10]$.

There is increasing recognition of the need to target HIV prevention efforts in Cameroon on females. Females in Cameroon are at increased risk of being infected because they initiate sexual activity at an earlier age than their male counterparts; they also tend to initiate sexual activity with older men who have higher infection rates than younger males [11,12]. In addition, many females engage in sexual relationships for economic benefits, due in part to the generally lower socio-economic status of women. In Cameroon men are more likely to complete high school, attend college and subsequently get better paying jobs than women. Women also tend to get married at younger ages than men. In addition, cultural norms dictate that the man is the head of the household and the decision-maker. All these factors translate to a subordinate role for women and the economic dependence of many females on their husband or sexual partners [1,12-14].

There is a paucity of HIV prevention intervention studies focusing on preadolescents or young adolescents in Cameroon. Although two intervention studies conducted in Cameroon have been reported [15,16], there are no known efforts to tailor HIV prevention interventions to be culturally sensitive, gender specific, and developmentally appropriate for use with Cameroonian preand young adolescents. Considering that most HIV prevention interventions were developed in the western world, assessment of the relevance and acceptability of such interventions strategies is key to maximizing their effectiveness for specific populations such as Cameroonian preadolescents. The aim of the research reported here was to assess the perceived relevance and accept- ability of an HIV prevention intervention to preadolescent Cameroonian females.

\section{Methods}

We analyzed post intervention data from a one group pretest posttest design study. The focus of the larger study was to determine the feasibility of recruiting and enrolling Cameroonian girls in HIV prevention studies, estimate the efficacy of the intervention, and assess cultural sensitivity of the intervention and study protocols. The findings reported here focus on the analysis of relevance and acceptability of the intervention to participants. Approval for the study was obtained from the primary author's institutional review board and the board of education of the school in Cameroon. In addition, parents provided written informed consent and participants provided written assent to participate in the study.

Setting and Sample: The study was conducted in a public school located in a university town which is also the capital of the South West Province of Cameroon. The town is also located in one of the provinces with the highest HIV/AIDS prevalence rates in Cameroon. All female students attending the school, aged 10 - 12, who could read and write, were considered eligible for the study. Sixty girls, who constituted $100 \%$ of those eligible, participated in the study.

Procedures: The study was advertized on school bulletin boards and flyers were distributed to all students attending the school. Consent forms were sent home with eligible students for their parents to complete. Transportation was provided to and from the school. The study was implemented on a Saturday so as to allow participants to participate in all school activities during weekdays.

All parental consent and student assent forms were collected and reviewed for completeness when student arrived at the study site. At the beginning of the day, participants completed a brief survey which included demographic characteristics and correlates of HIV risks in preadolescence. Participants took about 10 minutes to complete the questionnaire. Because the questionnaire focused on sensitive issues related to sexual behavior, strategies to encourage open and honest responses were implemented [17]. Strategies used in this study included: 1) explaining the importance of honest responses to questions because there were no right or wrong responses; 2) assuring participants that their responses would be kept confidential; 3) using code numbers instead of names on questionnaires; and 4) asking participants to deposit completed questionnaires in a sealed box instead of directly to the investigator.

The Intervention: The intervention was a day long workshop that was delivered in a group format. The 
workshop topics are outlined in Table 1. At the beginning of the intervention a lecture on bodily changes during puberty and HIV information such as nature of the $\mathrm{HIV}$ virus, modes of transmission and groups at risk in Cameroon was presented. The second part included specific units that focused on values clarification and building of specific HIV prevention behavioral skills. Each unit incorporated small group activities and a discussion. These units were completed in small groups of 10 students each. The investigator moved from group to group encouraging participation, answering questions, and facilitating activities. After each unit was completed, groups shared a summary of their discussions and activities with other participants and answered questions.

The intervention was built on the World Health Organization's (1994) Responsible Behavior: Delaying Sex curriculum, which allows content and protocols to be tailored to the specific needs, culture, and gender of the target population [18]. This curriculum includes a teacher's guide, students' guide, and a sample of questions that can be used to evaluate the curriculum. The primary investigator, a native of Cameroon, worked with local experts to adapt the intervention so that it matched the culture, age, and gender of participants. The intervention was extended with the addition of the theme: $B e$ Proud! Be Responsible! [19], to enhance personal, gender, and cultural pride. Activities in the intervention aimed at influencing attitudes, control beliefs, and behavioral norms about delaying sex, which are antecedents of intention and behavior. As part of the intervention participants were assisted in preparing planned culturally appropriate responses that could be used when they were faced with pressures to have sex. Examples of planned responses included refusing ("No, I am leaving”), delaying ("Let me think about it and we can talk again tomorrow"), and bargaining ("I won't do that but maybe we can do...”). Interactive activities that were also part of the intervention included: affirmations, life mapping, the use of role-plays, decision making in real-life scenarios, assertive communication scenarios, question and answer sessions, and group discussions (See Table 1)

Measures: A questionnaire administered prior to and immediately after the workshop intervention was used to measure demographic data and study variables. The relevance and acceptability of the intervention was assessed using 10 items developed specifically for use in the study with the help of a panel of seven Cameroonian health educators and teachers. The items were statements about the intervention that included understanding of the intervention, ease of reading the items, comfort with content, missing content, likelihood of recommending the intervention to others, and suggestions for modifying the intervention. For each item, participants completed

Table 1. Outline of intervention workshop curriculum guide.

\begin{tabular}{|c|c|c|}
\hline Section & $\begin{array}{c}\text { Content } \\
\text { Theme: Be proud! Be Responsible! }\end{array}$ & Target Variable \\
\hline Section 1 & $\begin{array}{l}\text { Lecture: Introduction, bodily changes during puberty, and HIV prevention } \\
\text { information Lecture }\end{array}$ & $\begin{array}{l}\text { Knowledge and } \\
\text { Attitudes }\end{array}$ \\
\hline Section 2 & HIV prevention: Delaying Sexual intercourse & \\
\hline Unit 1 & Activity: Discuss a variety of reasons for delaying sex until older or married & Attitudes and Norms \\
\hline Unit 2 & $\begin{array}{l}\text { Activity: Discussing the decisions to delay or not to delay: Why young people make } \\
\text { these decisions and the consequences, life mapping, and affirmations }\end{array}$ & $\begin{array}{l}\text { Norms and behavior } \\
\text { control }\end{array}$ \\
\hline Unit 3 & $\begin{array}{l}\text { Activity: Students practice responding to typical arguments that are used to pressure } \\
\text { girls to have sex }\end{array}$ & $\begin{array}{l}\text { Subjective norms and } \\
\text { behavior control }\end{array}$ \\
\hline Unit 4 & $\begin{array}{l}\text { Make students aware that they are not alone in delaying sex and providing guidelines } \\
\text { to assist students }\end{array}$ & Subjective norms \\
\hline Unit 5 & $\begin{array}{l}\text { Activity: Students practice guidelines for delaying sex in role-plays. Use guidelines } \\
\text { provided in previous unit. Facilitator communicate that delaying sex is not always } \\
\text { easy but specific behaviors skills can help }\end{array}$ & Behavior control \\
\hline Unit 6 & $\begin{array}{l}\text { Activity Expressing Affections without sex: Students discuss ways to demonstrate } \\
\text { affection that do not include sex. }\end{array}$ & Behavior control \\
\hline Unit 7 & Activity: Deciding how far to go ahead of time & Behavior control \\
\hline Unit 8 & Discuss passive, assertive, and aggressive behaviors & Behavior control \\
\hline Unit 9 & Activity: Learning to be assertive: students practice assertive communication & Behavior control \\
\hline Unit 10 & $\begin{array}{l}\text { Activity: Responding to persuasion: Using the Refuse, Delay, Bargain approach. } \\
\text { Student practice using this approach in role-plays }\end{array}$ & Behavior control \\
\hline Unit 11 & $\begin{array}{l}\text { Activity: Dealing with threats of violence: Discuss potential compromising situa- } \\
\text { tions, incentives for sex from older persons, threats of violence }\end{array}$ & Behavior control \\
\hline
\end{tabular}


two tasks. First, participants responded "yes” or "no" as to whether the item was true. Second, participants were asked to provide written comments about the aspect of the intervention addressed in the item. The items were administered immediately following completion of the intervention.

Data Management and Analysis: Descriptive statistics were used to summarize responses to sociodemographic items and the yes/no responses to the 10 items assessing the relevance and acceptability of the intervention. To explore differences in perceptions of appropriateness of the intervention, Fisher-exact tests were conducted to assess differences in responses to yes/no items in relation to differences in age, living conditions, educational level of parents, household income, and provinces or origin. The content of the written responses to each of the 10 items were analyzed and categorized into common themes related to the acceptability and relevance of the intervention

\section{Results}

The sociodemographic characteristics of participants are summarized in Table 2. The age of students ranged from $10-12$ years with a mean age of 10.5. The majority of participants lived with both parents (66.7\%). All participants reported they had not yet engaged in sexual activity. The literacy rate for participant's parents (98\%) was much higher than the national reported adult literacy rate of approximately $77 \%$ [20].

Participants hailed from eight different Englishspeaking areas of Cameroon, and the majority (85\%) was from English speaking provinces. Reported household incomes for the majority of participants (64\%) were higher than national median incomes and most students (61.7\%) had at least one member of their household who held a college degree.

The responses to the 10 items assessing the intervention are summarized in Table 3. The majority of the participants (78\%) reported favorable perceptions of the intervention. There were no significant differences in responses to the 10 items due to age or ethnicity. However a trend was noted in rating of the intervention content, with participants from the Norwest Province reporting less understanding of content (50\%) as compared to

Table 2. Sample characteristics $(\mathbf{N}=\mathbf{6 0})$.

\begin{tabular}{lll}
\hline Variable & $\mathrm{N}$ & $\%$ \\
\hline Age & 34 & \\
10 years old & 21 & 56.7 \\
11 years old & 5 & 35 \\
12 years old & & 8.3 \\
Living with & 40 & 66.7 \\
$\quad$ Both parents & 9 & 45 \\
$\quad$ One parent & 6 & 10 \\
$\quad$ Grand parents & 5 & 8.3 \\
$\quad$ Other relatives & & \\
Monthly Household Income & 20 & 33.3 \\
Below 50,000 CFA & 8 & 13.3 \\
50,000 CFA - 100,000 CFA & 12 & 20 \\
100,000 CFA - 200,000 CFA & 19 & 31.7 \\
Above 200,000 CFA & 1 & 1.7 \\
$\quad$ Unreported & 1 & 1.7 \\
Highest Educational level in Household & 4 & 6.7 \\
$\quad$ No Formal Education & 8 & 13.3 \\
Primary School & 6 & 10 \\
Secondary School & 37 & 61.7 \\
High School & 4 & 6.7 \\
$\quad$ University or Above & & \\
Other (Professional Diploma) &
\end{tabular}

Table 3. Summary of responses to relevance and acceptability items.

\begin{tabular}{lll}
\hline \multirow{2}{*}{ Item } & \multicolumn{2}{c}{ Frequency (Percentages) } \\
\cline { 2 - 3 } & Yes & No \\
\hline 1. Comfort with content & $44(73.3 \%)$ & $15(25 \%)$ \\
2. Study help change mind & $41(68.3 \%)$ & $12(28.3 \%)$ \\
3. Expected content not covered & $7(11.6 \%)$ & $52(86.7 \%)$ \\
4. Some content should be left out & $6.7(40.2 \%)$ & $52(86.7 \%)$ \\
5. Learned new skill & $44(73.3 \%)$ & $16(26.7 \%)$ \\
6. Would recommend study to others & $43(71.7 \%)$ & $16(26.7 \%)$ \\
\hline
\end{tabular}

(Note: Total sample was 60). 
participants from other provinces (81\% - 88\%); they were also less likely to agree to having learned new skills (60\%) than participants from other provinces (83\% 89\%) Twenty five percent of the participants reported not feeling comfortable with the topics covered in the intervention. However, participants failed to explain what specifically made them uncomfortable. Among those who provided explanations, their discomfort was associated with discussions of sexual behavior in general. Most participants indicated that the content of the intervention was sufficient. However, sixteen percent of the participants indicated that there was additional content that should be included in the intervention, including information about the progress being made toward the development of an HIV vaccine and current treatment modalities. A small portion of participants (6.7\%) indicated that certain content should be omitted however; they failed to provide specific information on what content this might be.

In general, most participants (78\%) reported that they had learned new skills, and would try to share skills they had learned with family members. They also indicated that they would recommend the intervention to others because they had learned new skills that would be useful to other people they knew.

\section{Discussion}

This was the first study that had directly assessed the relevance and acceptability of school-based HIV preventative interventions to preadolescents Cameroonian females. Considering that most HIV prevention interventions were developed for use with Western populations and do not target preadolescents, assessments of the relevance and acceptability of such interventions are warranted. In this study, most participants, including the youngest age group perceived the intervention appropriate. The impact of HIV/AIDS in Cameroon and the lack of age appropriate interventions that equip these students to protect themselves from the disease might have contributed to this positive perception. Participants were highly likely to have known at a personal level people who were suffering from or had died from the disease. To date, HIV preventative strategies in the country have focused almost exclusively on media campaigns promoting condom use and HIV testing. The relevance of the intervention to the target population likely resulted from the inclusion of strategies for protecting oneself from the disease. The importance preadolescent females and their parents attached to protection from the disease was evident in the $100 \%$ positive response of parents to allowing their children to take part in the study.

Findings in this study which suggest that the intervention was contextually appropriate are consistent with findings in other school-based studies in sub-Saharan Africa [21-24]. In these studies western change theories were integrated with qualitative information from local experts and the resulting interventions were perceived contextually relevant. In line with these studies, a number of steps were taken in the process of developing the intervention to ensure that students in Cameroon would be able to understand the content. Evaluation of intervention content and strategies by teachers involved in the educational system in Cameroon during the adaptation phase and the resulting modifications made from this evaluation contributed to the perceived appropriateness from even the youngest of participants. Furthermore, participants were aware that the primary investigator was native to the region, and this may have added to positive perceptions of the intervention.

When responses on appropriateness of the intervention were examined by age, no relationship was found between age and perceived appropriateness. It is worth noting that the majority of the participants (58.3\%) were 10 year-olds, and proportionately more 10 year-old participants than older participants thought the intervention was appropriate. The proportionately more favorable perceptions from 10 year-old students might be a developmental or a social desirability issue or simply due to chance since the difference among age groups were not significant.

The responses on appropriateness of the intervention were also examined by ethnicity. Participants were from two major English speaking ethnic regions or provinces of the country; the Northwest and Southwest provinces. A few of the participants (15\%) were from various other provinces in the country and were all grouped under "other". In general, students from all ethnic divisions considered the intervention and its components appropriate. However there were some non-significant differences between participants from different ethnic regions in their responses to some of the items concerning appropriateness of the intervention. More participants (81\%) from the Southwest province reported that they understood the content of the study than participants from the Northwest province (50\%) while more participants from the Northwest province (83\%) reported having learned new skills than participants from the Southwest province (60\%). These findings have to be interpreted with caution considering the convenience sample and the relatively small number of participants (20\%) from the Northwest province. These differences might be a reflection of variations in cultural norms related to sexuality issues between the two regions. In at least one descriptive study [10] conducted in the Northwest Province, researchers examining socio-cultural factors associated with risky sexual behaviors found differences among participants 
from different provinces which were attributed to differences in sexual permissiveness among ethnic groups. In the same study, participants from the Northwest Province were found to have initiated sexual activity earlier than their counterparts from other provinces [10]. Regional differences were also observed in a study on early initiation of sexual activity [25]. The percentage of women who were sexually active in adolescence and before marriage varied from $8 \%$ in some regions of the country to $69 \%$ in other regions [25]. Reports could not be found in the literature that provided any specific difference in cultural practices in these provinces that could account for the difference. Further exploration of these differences is warranted.

It must be noted that the choice of measures for appropriateness of the intervention might have influenced results obtained. The choice of a yes/no response format has the advantage of being more appealing to participants because it demands less concentration, but variability in the responses is also lost by using this format [26] The open-ended sections of the questions added to elicit more in depth information were often not used by participants. For those who used it, responses were often brief or cryptic and provided little to understanding participants' perceptions. Face to face interviews would have been a better approach in eliciting more in depth information. In addition because of the sensitive nature of the topic of the intervention, the age, and gender of participants the influence of social desirability bias cannot be ruled out.

In all, HIV infections pose a serious threat to the health of young Cameroonians. The prevalence rate continues to rise at an alarming rate in this population. Experts have identified equipping pre-adolescent females with skills necessary to reduce their vulnerability as an effective strategy towards reversing the epidemic. Evaluating the appropriateness of interventions to the developmental level, gender, and culture of participants is an essential step in developing effective and sustainable interventions. Findings in this study are encouraging and indicate that the intervention was appropriate from the participants' perspective. However these results need to be interpreted with caution because the small sample size and the convenience sampling approach used. In addition, there are limits to generalizability of the findings as the intervention was tailored based on knowledge of the developmental level and culture of participants. In spite of the limitations, findings and practical lessons learned from the study could inform other more rigorously designed HIV preventions interventions studies in Cameroon or studies targeting preadolescents.

\section{REFERENCES}

[1] Joint United Nations Program on HIV/AIDS, “AIDS Epidemic,” Update December 2009, Retrieved 21 January
2010.

http://data.unaids.org/pub/Report/2009/JC1700/Epi/Updat e/2009/en.pdf

[2] Joint United Nations Program on HIV/AIDS, "Epidemiological Fact Sheet on HIV and AIDS, Core Data on Epidemiology and Response, Cameroon,” 2008.

http://apps.who.int/globalatlas/predefinedReports/EFS200 8/full/EFS2008_CM.pdf

[3] United Nations Children's Fund, “Information on STI and HIV/AIDS."

www.unicef.org/wcaro/WCARO_Cameroon_Pub_KitPT ME-en.pdf

[4] C. Enah, “An Intervention to Increase Intentions and Behavioral Skills to Postpone Sexual Activity in Cameroonian Preadolescent Female Students," Dissertation Abstracts International 65/05, 2342B, 2004.

[5] C. Enah, M. Sommers, L. Moneyham, C. Long and G. Childs, "Piloting and HIV Prevention Intervention for Cameroonian Girls," Journal of Nurses in AIDS Care, Vol. 21, No. 6, 2010, pp. 512-521. doi:10.1016/j.jana.2010.02.011

[6] World Health Organization, "HIV/AIDS Epidemiological Surveillance Report for the WHO African Region,” 2007. http://www.afro.who.int/aid/publications/index.html

[7] World Health Organization, "2006 AIDS Epidemic Update," 2006.

http://www.who.int/hiv/mediacentre/04_sub_saharan_Afr ica_2006_EpiUpdate_eng.pdf

[8] G. Burkholder, L. Harlow and J. Washkwich, "Social Stigma, HIV/AIDS Knowledge, and Sexual Risk,” Journal of Applied Biobehavioral Research, Vol. 4, No. 1, 1999, pp. 27-44.

doi:10.1111/j.1751-9861.1999.tb00053.x

[9] P. T. Tebon, "Cameroon National Composite Policy Index Report 2008,” 2008.

http://data.unaids.org/pub/report/2008/Cameroon_2008_n cpi en.pdf

[10] M. Sommer, “An Overlooked Priority: Puberty in Sub-Saharan Africa," American Journal of Public Health, Vol. 101, No. 6, 2011, pp. 979-981. doi:10.2105/AJPH.2010.300092

[11] Joint United Nations Program on HIV/AIDS, "Report on the HIV/AIDS Epidemic,” 26 February 2007. http: www.unaids.html

[12] M. Rwenge, "Sexual Risk Behaviors among Young People in Bamenda, Cameroon," International Family Planning Perspectives, Vol. 26, No. 3, 2000, pp. 188-123, 130.

[13] D. Meekers and A. Calves, "Main Girlfriends, Girlfriends, Marriage, and Money: The Social Context of HIV Risk Behavior in Sub-Saharan Africa," Health Transitions Review, Vol. 7 (Supplement), 1997, pp. 361-375.

[14] N. Lydie, N. Robinson, B. Ferry, E. Akam, M. De Loenzien, L. Zekeng and S. Abega, "Adolescent Sexuality in Yaoundé, Cameroon,” Journal of Biosocial Sciences, Vol. 36, No. 5, 2004, pp. 597-616. 
[15] J. Speizer, B. Taambashe and S. Tegang, “An Evaluation of the 'Entre Nous Jeunes' Peer-Educator Program or Adolescents in Cameroon,” Studies in Family Planning, Vol. 32, No. 4, 2001, pp. 339-351. doi:10.1111/j.1728-4465.2001.00339.x

[16] D. Meekers, S. Agha and M. Klein, "The Impact on Condom Use of the '100\% Jeune' Social Marketing Program in Cameroon,” Journal of Adolescent Health, Vol. 36, No. 6, 2005, p. 530. doi:10.1016/j.jadohealth.2004.10.012

[17] B. Mensch, P. Hewett and A. Erulkar, "The Reporting of Sensitive Behavior among Adolescents: A Methodological Experiment in Kenya,” Demography, Vol. 40, No. 2, 2003, pp. 247-268. doi:10.1353/dem.2003.0017

[18] World Health Organization, "Responsible behavior: Delaying Sex,” In Curriculum Planners’ Handbook, WHO/ UNESCO, Geneva, 1994.

[19] L. Jemmott, J. Jemmott and K. McCaffee, "Be Proud! Be Responsible! Strategies to Empower Youth to Reduce Their Risk of AIDS," Columbia University School of Nursing, New York, 1996.

[20] World Health Organization, "2006 AIDS Epidemic Update," 2006.

http://www.who.int/hiv/mediacentre/04 sub saharan Afr ica_2006_EpiUpdate_eng.pdf

[21] J. Jemmott, L. Jemmott, A. O’Leary, Z. Ngwane, L. Icard, S. Bellamy, et al., "School-Based Randomized Controlled trial of an HIV/STD Risk Reduction Intervention for
South African Adolescents," Archives of Pediatric Medicine, Vol. 164, No. 10, 2010, pp. 923-929. doi:10.1001/archpediatrics.2010.176

[22] A. Karnell, P. Cupp, R. Zimmerman, S. Feist-Price and T. Bennie, "Efficcacy of an American Alcohol and HIV Curriculum Adapted for Use in Aouth Africa: Results of a Pilot Study in Five Townships," AIDS Education and Prevention, Vol. 18, No. 4, 2006, pp. 295-310. doi:10.1521/aeap.2006.18.4.295

[23] M. Fitzgerald, B. Stanton, N. Terreri, H. Shipena, X. Li, J. Kahihuata, et al., "Use of Western-Based HIV RiskReduction Interventions Targeting Adolescents in an African Setting," Journal of Adolescent Health, Vol. 25, 1999, pp. 52-61. doi:10.1016/S1054-139X(98)00120-7

[24] K. Klepp, S. Ndeki, M. Leshabari, P. Hannan and B. Lyimo, "AIDS Education in Tanzania: Promoting Risk Reduction among Primary School Children," American Journal of Public Health, Vol. 87, No. 12, 1997, 1931-1936. doi:10.2105/AJPH.87.12.1931

[25] B. Kuate-Defo, "Premarital Sexual Intercourse during Adolescence: Trends and Determinants of Regional Differences,” In: B. Kuate-Defo, Ed., Sexuality and Reproductive Health during Adolescence in Africa with Special Reference to Cameroon, University of Ottawa Press, Ottawa, 1998, pp. 121-140.

[26] R. Devellis, "Scale Development: Theory and Applications,” Sage Publications, Newbury Park, 1991. 